\title{
The symmetry energy from heavy ion reactions
}

\author{
W. Trautmann* \\ GSI Helmholtzzentrum für Schwerionenforschung GmbH, D-64291 Darmstadt, Germany \\ E-mail: w.trautmann@gsi.de
}

The equation of state of asymmetric nuclear matter is an essential ingredient in nuclear physics and astrophysics but, as of today, insufficiently constrained by experiment. The symmetry energy, i.e. the difference between the energy of neutron matter and of symmetric matter, and its dependence on density are important for the modelling of neutron stars and supernova explosions as well as for isospin phenomena in nuclear reactions and nuclear structure. Heavy-ion reactions are a unique tool for obtaining information on the symmetry energy in the laboratory, and a considerable activity is presently devoted to its behavior at sub- and supra-saturation density.

In this contribution, new results are presented which were obtained in experiments conducted at the GSI laboratory with relativistic heavy-ion beams. A systematic study of isotopic effects in spectator fragmentation was performed at the ALADIN spectrometer with ${ }^{124} \mathrm{Sn}$ primary and ${ }^{107} \mathrm{Sn}$ and ${ }^{124} \mathrm{La}$ secondary beams of $600 \mathrm{MeV} /$ nucleon incident energy. The analysis within the Statistical Fragmentation Model shows that the symmetry-term coefficient entering the liquiddrop description of the emerging fragments decreases significantly as the multiplicity of fragments and light particles from the disintegration of the produced spectator systems increases. Higher densities were probed in the FOPI/LAND study of nucleon and light-particle flows in central and mid-peripheral collisions of ${ }^{197} \mathrm{Au}+{ }^{197} \mathrm{Au}$ nuclei at $400 \mathrm{MeV} /$ nucleon incident energy. From the comparison of the measured neutron and hydrogen squeeze-out ratios with predictions of the UrQMD model a moderately soft symmetry term with a density dependence of the potential term proportional to $\left(\rho / \rho_{0}\right)^{\gamma}$ with $\gamma=0.9 \pm 0.3$ is favored.

XLVIII International Winter Meeting on Nuclear Physics, BORMIO2010

January 25-29, 2010

Bormio, Italy

${ }^{*}$ Speaker. 


\section{Introduction}

The symmetry energy is quite familiar from the Bethe-Weizsäcker semi-empirical mass formula. The negative contribution $B_{\mathrm{sym}}=-23.7 \mathrm{MeV} \cdot(N-Z)^{2} / A$ to the binding energy is responsible for the parabolic increase of the masses at either side of the valley of stability. Its competition with the Coulomb term determines the neutron richness of heavy nuclei and, e.g., a reduced symmetry term would allow much heavier nuclei to exist. This last point, in fact, represents already one of the many connections of the symmetry energy with astrophysical phenomena [1].

More elaborate mass formulae distinguish between volume and surface contributions to the symmetry energy, reflecting the reduction of symmetry effects at lower densities [2, 3]. If this is investigated with the Fermi-gas model by expanding the expression for the nucleon kinetic energies in powers of $(N-Z)$, the symmetry energy is found to increase in proportion to $\rho^{2 / 3}$, with $\rho$ representing the nuclear density. However, the coefficient in the obtained expression $B_{\mathrm{sym}}=$ $-e_{F} / 3 \cdot(N-Z)^{2} / A$ is only about $10 \mathrm{MeV}\left(e_{F}\right.$ is the Fermi energy). The kinetic part thus amounts to only about $1 / 3$ of the observed effect. The remaining $2 / 3$ are contributed by the nuclear interactions.

Regarding the equation of state of nuclear matter, the dependence of the energy per nucleon $E_{A}$ on the density $\rho$ and asymmetry $\delta=\left(\rho_{n}-\rho_{p}\right) / \rho$ (with the neutron and proton densities $\rho_{n}$ and $\left.\rho_{p}\right)$ is usually expressed as $E_{A}=E_{A}(\rho, \delta=0)+E_{\text {sym }}(\rho) \cdot \delta^{2}$. The quadratic approximation has been shown to be quite realistic and higher order terms are found to be small [4,5]. At saturation, $E_{\text {sym }}\left(\rho=\rho_{0}\right)$ is of typically $30 \mathrm{MeV}$, corresponding to the volume-symmetry coefficient in mass formulae (e.g., 28.1 MeV in that of Myers and Swiatecki [2]). When discussing the density dependence, two regimes have to be distinguished. At densities lower than the saturation density and at temperatures below the critical temperature of about $18 \mathrm{MeV}$, nuclear matter is in the mechanically unstable region of the nuclear liquid-gas phase transition, and the formation of fragments has to be taken into account. Even at the very low densities appearing in the envelopes of super nova explosions, clustering is important and the symmetry energy remains at a finite value [6]. Microscopic models, without the effect of clustering, are rather consistent in their predictions for the symmetry energy below saturation with a density dependence of about $\rho^{2 / 3}$ [7]. Very different predictions are obtained at supra-saturation densities, ranging from the asy-soft behavior of the Gogny force to asy-stiff density dependences of up to $\rho^{2}$. A useful quantity to distinguish these cases is the parameter $L=3 \rho_{0} \cdot \mathrm{d} E_{\mathrm{sym}} /\left.\mathrm{d} \rho\right|_{\rho_{0}}$ which is proportional to the slope of the symmetry term at the saturation point. $L \approx 20 \mathrm{MeV}$ characterizes a soft and $L \approx 100 \mathrm{MeV}$ a stiff density dependence.

On the experimental side, considerable progress has been made with experiments testing the strength of the symmetry term at densities near or below saturation. This includes isospin diffusion and neutron/proton yield ratios [8] as well as the isospin dependence of the isoscalar giant monopole resonance [9] or the strength of the pygmy resonance in neutron rich nuclei [10]. Quite consistent results of $\gamma \approx 0.7$ corresponding to $L=40-70 \mathrm{MeV}$ have been reported [8]. In the statistical analysis of multi-fragmentation reactions, the symmetry term in the mass formula used for the fragment description is tested. Since the fragments are not isolated but rather emerging from the hot environment of the collision zone their properties may be modified (Section 2). One of the motivations of these studies is the overlap of the freeze-out conditions with those encountered in supernova scenarios, permitting the study of stellar processes in the laboratory [1]. 
To probe supra-saturation densities, central collisions of heavy ions at sufficiently high energies are required. Collective flow observables and production ratios of produced mesons have been proposed as probes for the early dense reaction phases [11,12]. The investigation of particle ratios at energies close to threshold has so far not led to conclusive results. The ratios of $\mathrm{K}^{+} / \mathrm{K}^{0}$ turned out to be less sensitive to the symmetry energy in actual collisions than predicted for equilibrium conditions [13]. Variations of up to $20 \%$ between the soft and stiff cases are expected for $\pi^{-} / \pi^{+}$ ratios. However, the competition of mean-field and collision effects is, apparently, very delicate. Different predictions leading to opposite conclusions have been obtained from the comparison of the FOPI data for ${ }^{197} \mathrm{Au}+{ }^{197} \mathrm{Au}$ collisions at $400 \mathrm{MeV} /$ nucleon [14] with different models $[15,16]$. The study of flow effects has only started and first results will be shown in Section 3.

\section{Isotopic effects in projectile fragmentation}

Isotopic effects in projectile multi-fragmentation at relativistic energies were studied with experiment S254 of the ALADIN collaboration, conducted in 2003 at the SIS heavy-ion synchrotron [17]. Besides stable ${ }^{124} \mathrm{Sn}$ beams, neutron-poor secondary ${ }^{107} \mathrm{Sn}$ and ${ }^{124} \mathrm{La}$ beams provided by the FRS fragment separator, all with $600 \mathrm{MeV} /$ nucleon incident energy, were used in order to explore a wide range of isotopic compositions.

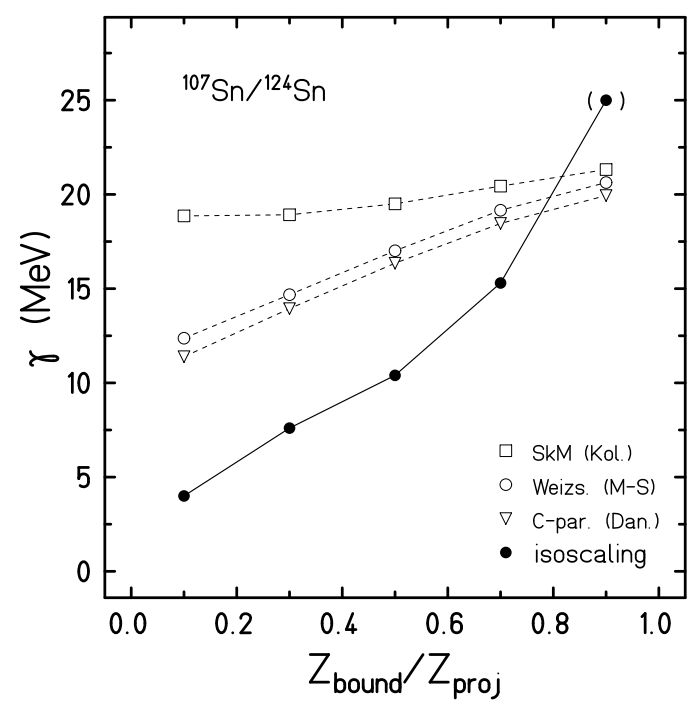

Figure 1: Symmetry term coefficient $\gamma$ as expected from the changing fragment-mass distributions using surface and volume symmetry-term coefficients from Refs. [2, 3, 21] (open circles, triangles, and squares, respectively) in comparison with the coefficient $\gamma$ for hot fragments obtained from the isoscaling analysis with the Statistical Multifragmentation Model (dots).

The comparison of the measured fragment yields from neutron-rich and neutron-poor systems shows that isoscaling is observed [18]. The isoscaling parameter $\alpha$ is found to decrease rapidly as the violence of the collision increases and larger numbers of fragments and light particles are produced, confirming earlier results for the fragmentation of target spectators in reactions of ${ }^{12} \mathrm{C}$ on ${ }^{112,124} \mathrm{Sn}$ at 300 and $600 \mathrm{MeV} /$ nucleon [19]. The analysis of the data with the Statistical Multifragmentation Model [20] was performed with ensemble calculations adapted to the participantspectator scenario at relativistic energies. An ensemble of excited systems with varying excitation 
energy and mass was chosen so as to best reproduce the charge spectra and correlations observed for the fragment production.

The symmetry-term coefficient $\gamma$ for hot fragments resulting from the isoscaling analysis for the ${ }^{107,124} \mathrm{Sn}$ pair of reactions is shown in Fig. 1 as a function of $Z_{\text {bound }} / Z_{\text {proj. }}$. The sorting variable $Z_{\text {bound }}=\Sigma Z_{i}$ with $Z_{i} \geq 2$ represents approximately the charge of the spectator system and is inversely correlated with the transferred excitation energy. For comparison, several predictions are shown in the figure which contain the effect of the surface-symmetry term as it is used in mass formulae fitted to ground-state masses [2,21] or to the energies of isobaric analog states [3]. For the five bins in $Z_{\text {bound }}$, the experimental $Z$ distributions were converted to mass distributions, using the projectile $N / Z$, and the effective symmetry energy averaged over the set of partitions was calculated. The smaller fragments produced at higher excitations cause the effective mean symmetry term to decrease with decreasing $Z_{\text {bound }}$ but at a slower rate than that resulting from the isoscaling analysis of the measured isotope yield ratios.

\section{Collective flows}

In two experiments at GSI combining the LAND and FOPI (Phase 1) detectors, both neutron and hydrogen collective flow observables from ${ }^{197} \mathrm{Au}+{ }^{197} \mathrm{Au}$ collisions at 400, 600, and 800 $\mathrm{MeV} /$ nucleon have been measured [22]. This data set is presently being reanalyzed in order to determine optimum conditions for a dedicated new experiment [23], but also with the aim to produce constraints for the symmetry energy at the high densities probed in central collisions [24]. The results reported here are obtained by comparing with predictions of the UrQMD model which has recently been adapted to heavy ion reactions at intermediate energies [25].

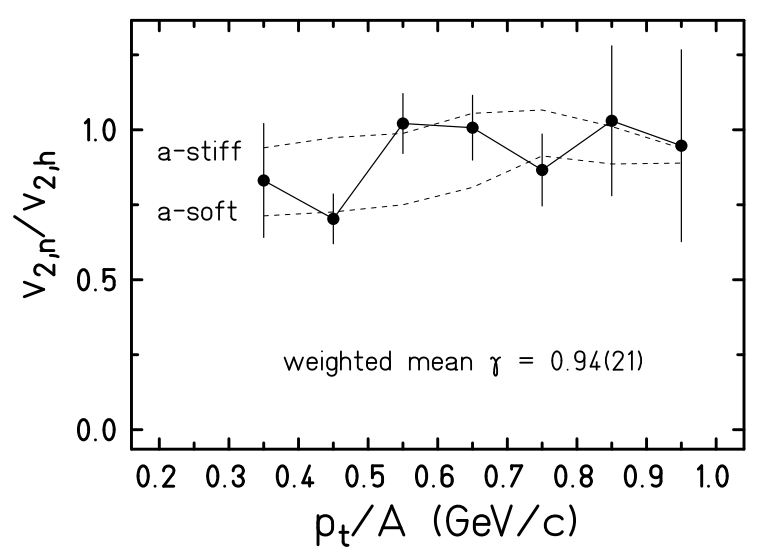

Figure 2: Ratio of elliptic flow parameters $v_{2, n}$ for neutrons and $v_{2, h}$ for hydrogen isotopes (dots) for central $(b<7.5 \mathrm{fm})$ collisions of ${ }^{197} \mathrm{Au}+{ }^{197} \mathrm{Au}$ at $400 \mathrm{MeV} /$ nucleon as a function of the transverse momentum per nucleon $p_{t} / A$ in comparison with the UrQMD predictions for $\gamma=1.5$ (a-stiff) and $\gamma=0.5$ (a-soft) represented by the dashed lines.

The UrQMD predictions were calculated for two values of the power-law exponent describing the density dependence of the potential part of the symmetry energy, $\gamma=1.5$ (asy-stiff) and $\gamma=0.5$ (asy-soft) and filtered with the acceptance of the FOPI/LAND experiment. For the quantitative evaluation, the ratio of neutron-over-hydrogen elliptic flows is proposed as an observable which 
should be insensitive to uncertainties resulting from the experimental determination of the reaction plane and from the matching of the experimental and theoretical impact-parameter intervals. The comparison shows that the experimental ratios scatter in-between the predictions for the asy-soft and asy-stiff cases (Fig. 2). A linear interpolation, averaged over $0.3<p_{t} / A \leq 1.0 \mathrm{GeV} / \mathrm{c}$, yields $\gamma=0.94 \pm 0.21$. Together with the kinetic term proportional to $\left(\rho / \rho_{0}\right)^{2 / 3}$, the squeeze-out data indicate a moderately soft behavior of the symmetry energy at supra-saturation densities.

The elliptic flow of nucleons and light particles thus promises to serve as a useful probe. It is clear, however, that new data of higher statistical significance will be needed in order to confirm the present result and to permit more precise tests of the consistency of model descriptions. In the future, sufficiently intense beams from radioactive beam facilities can be expected to further enlarge the experimental possibilities.

Illuminating discussions with Lie-Wen Chen, Bao-An Li, M. Di Toro, and H.H. Wolter are gratefully acknowledged. This work has been supported by the European Community under contract No. HPRI-CT-1999-00001 and FP7-227431 (HadronPhysics2).

\section{References}

[1] A.S. Botvina and I.N. Mishustin, Phys. Lett. B 584 (2004) 233; Nucl. Phys. A 843 (2010) 98.

[2] W.D. Myers and W.J. Swiatecki, Nucl. Phys. 81 (1966) 1.

[3] P. Danielewicz and J. Lee, Int. J. Mod. Phys. E 18 (2009) 892.

[4] U. Lombardo and W. Zuo, in Isospin Physics in Heavy-Ion Collisions at Intermediate Energies, ed. by B.-A. Li and W.U. Schröder, Nova Science, New York, 2001, p. 1.

[5] Bao-An Li, Lie-Wen Chen, Che Ming Ko, Phys. Rep. 464 (2008) 113.

[6] H.H. Wolter, contribution in this volume.

[7] M. Baldo et al., Nucl. Phys. A 736 (2004) 241.

[8] M.B. Tsang et al., Phys. Rev. Lett. 102 (2009) 122701.

[9] T. Li et al., Phys. Rev. C 81 (2010) 034309.

[10] A. Klimkiewicz et al., Phys. Rev. C 76 (2007) 051603(R).

[11] Bao-An Li, Phys. Rev. Lett. 88 (2002) 192701.

[12] G. Ferini et al., Nucl. Phys. A 762 (2005) 147.

[13] X. Lopez et al., Phys. Rev. C 75 (2007) 011901(R).

[14] W. Reisdorf et al., Nucl. Phys. A 781 (2007) 459.

[15] Z. Xiao et al., Phys. Rev. Lett. 102 (2009) 062502.

[16] Z.-Q. Feng and G.-M. Jin, Phys. Lett. B 683 (2010) 140.

[17] C. Sfienti et al., Phys. Rev. Lett. 102 (2009) 152701.

[18] S. Bianchin et al., in Proceedings of the XLV International Winter Meeting on Nuclear Physics, Bormio, Italy, ed. I. Iori and A. Tarantola, (Ricerca Scientifica ed Educazione Permanente Suppl., vol. 127, Milano, 2007), p. 233.

[19] A. Le Fèvre et al., Phys. Rev. Lett. 94 (2005) 162701.

[20] J.P. Bondorf et al., Phys. Rep. 257 (1995) 133.

[21] V.M. Kolomietz and A.I. Sanzhur, Eur. Phys. J. A 38 (2008) 345.

[22] Y. Leifels et al., Phys. Rev. Lett. 71 (1993) 963;

D. Lambrecht et al., Z. Phys. A 350 (1994) 115.

[23] R.C. Lemmon et al., proposal for SIS experiment S394 (2009, unpublished).

[24] W. Trautmann et al., preprint arXiv:1001.3867 (2010).

[25] Q. Li et al., J. Phys. G 31 (2005) 1359. 